\title{
Nonlinear localized modes at phase-slip defects in waveguide arrays
}

\author{
Mario I. Molina ${ }^{1,2}$ and Yuri S. Kivshar ${ }^{2}$ \\ ${ }^{1}$ Departmento de Física, Facultad de Ciencias, Universidad de Chile, Santiago, Chile \\ ${ }^{2}$ Nonlinear Physics Center, Research School of Physical Sciences and Engineering, \\ Australian National University, Canberra ACT 0200, Australia
}

We study light localization at a phase-slip defect created by two semi-infinite mismatched identical arrays of coupled optical waveguides. We demonstrate that the nonlinear defect modes possess the specific properties of both nonlinear surface modes and discrete solitons. We analyze stability of the localized modes and their generation in both linear and nonlinear regimes.

(C) 2018 Optical Society of America

OCIS codes: $060.4370 ; 190.4350 ; 190 ; 6135$

The study of nonlinear dynamics in discrete systems has attracted a special attention recently due to novel physics and possible interesting applications ${ }^{1}$. In particular, it is well known that discrete photonic systems can support different types of spatially localized states in the form of discrete solitons 1,2 . These solitons can be controlled by the insertion of suitable defects in an array, as was suggested theoretically ${ }^{3,4}$ and also verified experimentally for arrays of optical waveguides 5 . Defects may provide an additional physical mechanism for light confinement, and they can support both linear and nonlinear localized modes, which has been studied theoretically for different nonlinear models $\frac{6,7,8}{}$ and observed experimentally in one-dimensional photonic lattices ${ }^{9}$.

In this Letter, we introduce a novel type of nonlinear defect in waveguide arrays closely linked with the recently discussed phase-slip defects in two-dimensional photonic crystals 10,11. In particular, we demonstrate that two semi-infinite mismatched identical arrays of optical waveguides can support a variety of linear and nonlinear localized modes with the specific properties of both discrete solitons ${ }^{1}$ and nonlinear surface modes ${ }^{12,13}$. We analyze stability of the localized modes and their generation in both linear and nonlinear regimes.

We consider an array of nonlinear optical waveguides created by two semi-infinite identical mismatched arrays, as shown in Fig. 1. In this array, two mismatched waveguides at the sites $n=m$ and $n=m+1$ interact with a different coupling parameter, $V^{\prime} \neq V$, so that the coupledmode system can be described by the discrete equations for the normalized mode amplitudes $E_{n}$,

$$
\begin{gathered}
i \frac{d E_{n}}{d z}+V\left(E_{n+1}+E_{n-1}\right)+\sum_{m} V_{n m} E_{m}+\gamma\left|E_{n}\right|^{2} E_{n}=0 \\
\sum_{m} V_{n m} E_{m}=\left(V^{\prime}-V\right)\left[\delta_{n, m} E_{m+1}+\delta_{n, m+1} E_{m}\right],
\end{gathered}
$$

where $E_{n}$ are defined in terms of the actual electric fields $\mathcal{E}_{n}$ as $E_{n}=\left(2 \lambda_{0} \eta_{0} / \pi n_{0} n_{2}\right)^{1 / 2} \mathcal{E}_{n}$, where $\lambda_{0}$ is the freespace wavelength, $\eta_{0}$ is the free-space impedance, $n_{2}$ and $n_{0}$ are nonlinear and linear refractive indices of each waveguide, and $\gamma( \pm 1)$ defines the type of nonlinearity.

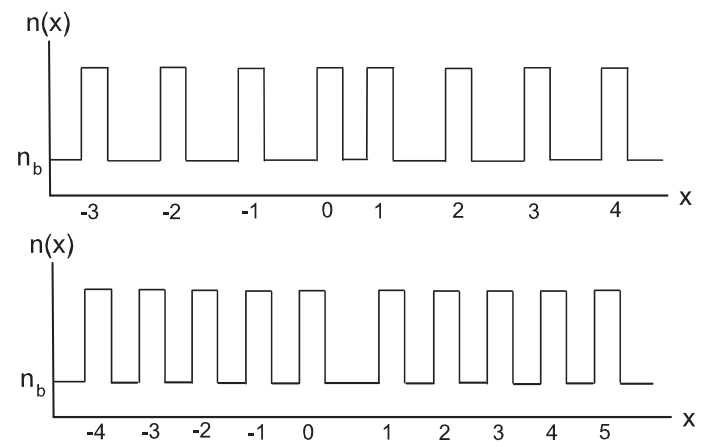

Fig. 1. Transverse profile of the refractive index for an array of weakly coupled nonlinear optical waveguides with a phase-slip defect located between the waveguides at the sites $m=0$ and $m=1$. Top: $V^{\prime}>V$, bottom: $V^{\prime}<V$.

We look for stationary solutions of Eqs. (1), (2) in the form $E_{n}(z)=E_{n} \exp (i \beta z)$, and consider first the linear waveguide array (or the limit of low beam powers) when $\gamma=0$. In this case, we expect that localized modes may exist for $V^{\prime}>V$ only, since decreasing the ratio $V^{\prime} / V$ decouples the chain into two pieces, and each of the semiinfinite chains does not support surface modes ${ }^{12,13}$. We search for localized solutions of the form $E_{n}=A \xi^{|n-m|}$, for $n \leq m$, and $E_{n}=B \xi^{(n-m-1)}$, for $n \geq m+1$, where $|\xi|<1$. After some algebra, we obtain $\beta=V[\xi+(1 / \xi)]$, $B / A=\xi\left(V^{\prime} / V\right)$ and $\xi= \pm\left|V / V^{\prime}\right|$ so that indeed, localized modes require the condition $V^{\prime}>V$.

For $\xi=\left|V / V^{\prime}\right|$ and $A=B$ we obtain unstaggered localized modes [see Fig. 2(a)]: $E_{n}=A\left|V / V^{\prime}\right|^{|n-m|}$, for $n \leq m$, and $E_{n}=A\left|V / V^{\prime}\right|^{n-m-1}$, for $n \geq m+1$ with the propagation constant $\beta / V=\left|V / V^{\prime}\right|+\left|\bar{V}^{\prime} / V\right|$. Similarly, for $\xi=-V / V^{\prime}$ and $A=-B$ we obtain the corresponding staggered localized modes [see Fig. 2(b)].

Next, we consider the nonlinear case described by the stationary form of Eqs. (1), (2) for $\gamma>0$. For a given value of $\beta$, the system of the stationary equations is solved numerically by a multidimensional Newton- 

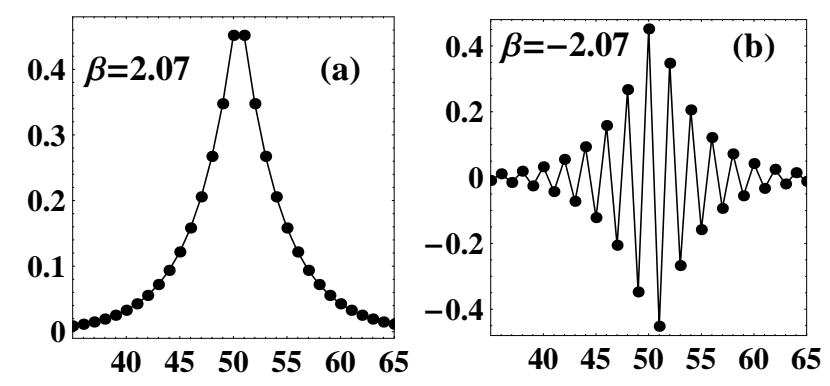

Fig. 2. Linear unstaggered (a) and staggered (b) localized modes at the phase-slip defect for $V^{\prime} / V=1.3(N=$ $100, m=50)$.

Raphson scheme. As we are interested in the modes localized near the defect, we look for the modes with the mode maxima near the slip boundary that decay quickly away from the bond impurity. We find that the results vary depending on whether $V^{\prime}>V$ or $V^{\prime}<V$.

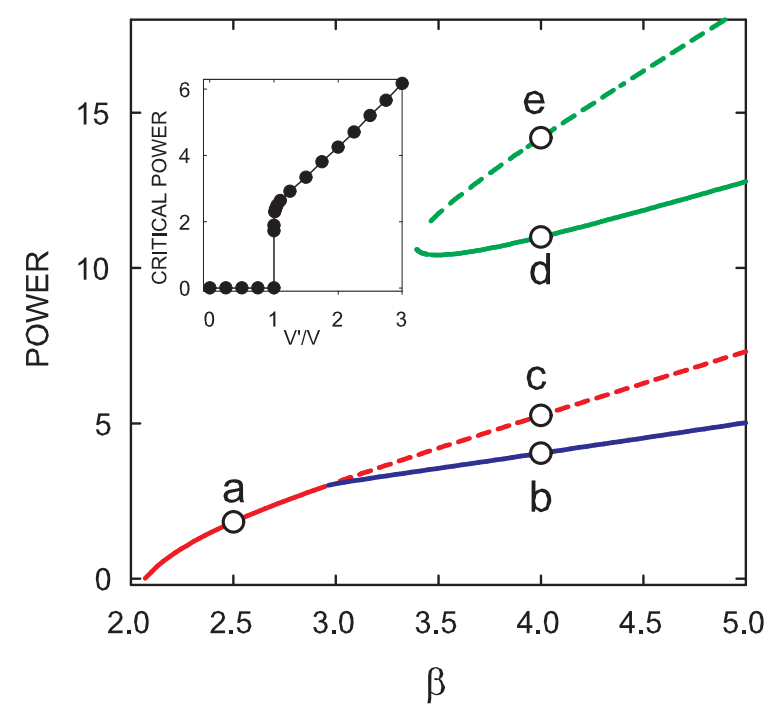

Fig. 3. Power vs. propagation constant for several families of nonlinear localized modes at the phase-slip defect for $V^{\prime} / V=1.3$. Solid (dashed) curves denote stable (unstable) branches. Inset: Minimum power to destabilize the fundamental mode vs. coupling mismatch.

Figures 3 and 4 depict the mode power vs. propagation constant and show specific examples of the mode profiles, for the case $V^{\prime}>V$. First, one of the nonlinear modes extends all the way down to zero power, and it generalizes the linear mode found above [see the curve (a-c) in Fig. 3 and Figs. $4(a, c)]$. This mode becomes unstable above a certain threshold power, and it transforms into an odd mode centered at any of the two equivalent sites coupled

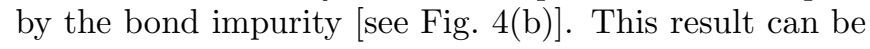

easily understood from the known instability of an even mode for a discrete homogeneous lattice: As the power is increased, the effective coupling is decreased and the distinction between $V$ and $V^{\prime}$ becomes blurred. In the high-power limit, the even-like localized state "feels" as inside an homogeneous lattice; hence the onset of instability. The inset in Fig. 3 3 shows the critical power needed to destabilize the fundamental, even-like localized mode. The most interesting feature of this curve is that it rises very steeply as soon as the ratio $V^{\prime} / V$ is slightly above one, followed by a slow, almost linear-like growth. This suggests that a tiny amount of mismatch is enough to stabilize the even-like mode at the phase slip.
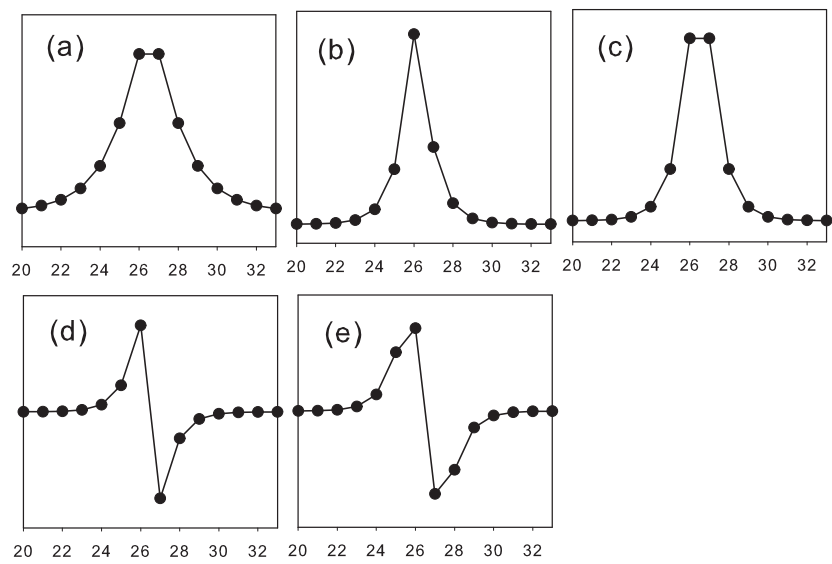

Fig. 4. (a-e) Examples of the nonlinear localized modes marked by the letters a,b,c,d, and e in Fig. 3.

For larger powers, we find novel types of nonlinear modes localized at the phase-slip defect [see Figs. [4(d,e)] resembling a bound state of two simpler modes. These modes resemble the so-called twisted modes found earlier in the homogeneous chain $\stackrel{7,14}{ }$, and they exist only above a certain power threshold. The complementary unstable mode looks like the twisted mode with "shoulders" [See Fig. 4(e)].

In the case of a weaker bond defect, i.e. $V^{\prime}<V$, linear localized modes do not exist. This result is consistent with the case of surface modes 12,13 where a certain power threshold is required to support a mode localized at the edge of the waveguide array. Similarly, in this model the localized mode appear for finite powers (see the branch marked 'a' in Fig. (5). The mode profiles are similar to those presented in Figs. 4(a-e). In this case, all localized modes are strictly nonlinear i.e., they disappear in the limit $\gamma \rightarrow 0$. As a result, most of those modes are unstable, and only two modes corresponding to the lower power are stable.

We should mention that all modes for $\gamma>0$ remain unstaggered, and staggered modes in this model appear only for $\gamma=-1$, being found through a simple transformation $E_{n} \rightarrow(-1)^{n} E_{n}$ applied to all types of modes discussed above. 


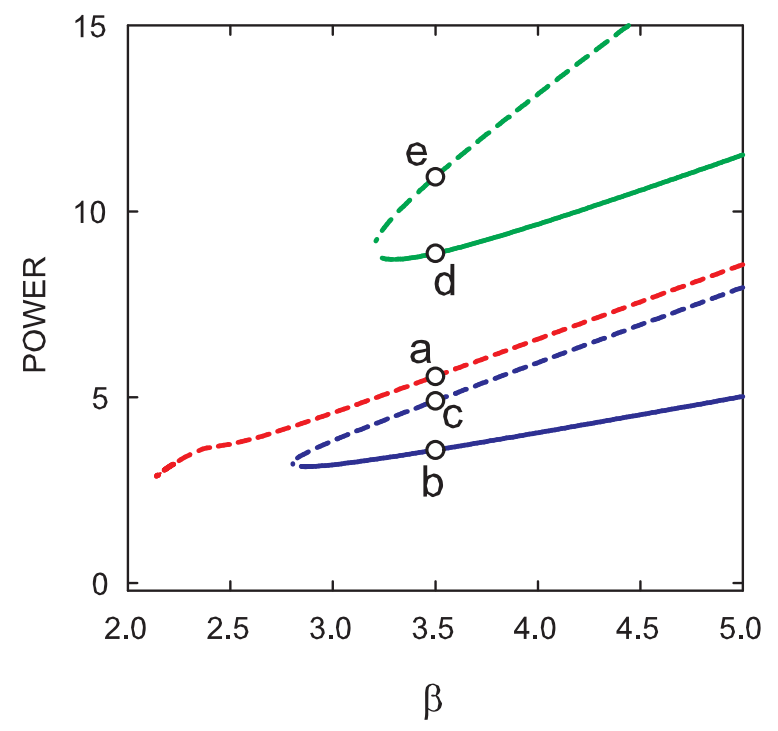

Fig. 5. Power vs. propagation constant for several localized states at the phase-slip defect for $V^{\prime} / V=0.7$. Solid (dashed) lines denote stable (unstable) branches.

Finally, we analyze the generation of the defect modes by an input beam sent to one of the waveguides of the phase-slip defect. For weaker coupling $\left(V^{\prime}<V\right)$ and low powers, we observe no power localization near the defect sites, and the input power diffracts as in homogeneous arrays [see Fig. 6(a)]. However, when we increase the input power, we are able to generate the asymmetric nonlinear defect mode that correspond to the lowest branch ( marked with 'b') in Fig. 5 and finite powers, similar to the excitation of discrete surface solitons ${ }^{12.13}$. On the contrary, the surface mode is always generated for the case of stronger coupling, $V^{\prime}>V$, when the defect mode exists in the linear regime, as shown in Figs. $6(\mathrm{c}, \mathrm{d})$.

In conclusion, we have introduced and described novel types of nonlinear defect modes localized at a phaseslip defect in an array of nonlinear optical waveguides. We have demonstrated that these localized modes possess many specific properties of both discrete solitons and nonlinear surface modes, and they can be easily generated in both linear and nonlinear regimes.

This work has been supported by Fondecyt grants 1050193 and 7050173 in Chile, and by the Australian Research Council in Australia. M.I.M. thanks the Nonlinear Physics Center at the Australian National University for hospitality and financial support.

\section{References}

1. See, e.g., D.N. Christodoulides, F. Lederer, and Y. Silberberg, Nature 424, 817 (2003) and references therein.

2. Yu. S. Kivshar and G. P. Agrawal, "Optical Solitons: From Fibers to Photonic Crystals" (Academic Press,

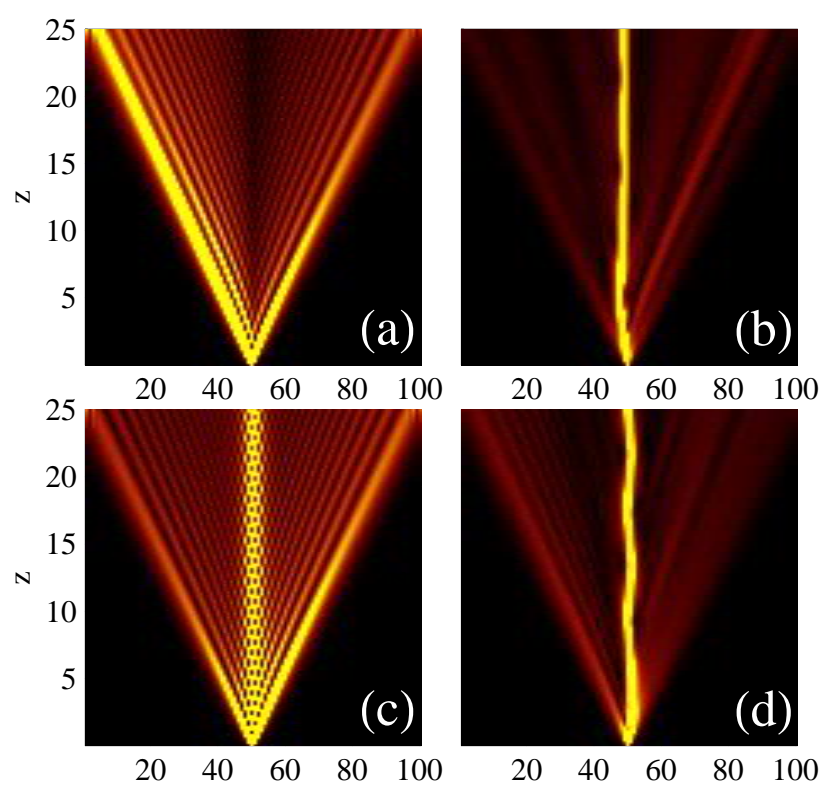

Fig. 6. One-waveguide excitation of the defect modes. Top: $V^{\prime} / V=0.7$, i.e. for a weak bond defect, for the input amplitude (a) $V_{0}=1.0$ and (b) $V_{0}=2.0$ (b). Bottom: same as above but for stronger bond defect, $V^{\prime} / V=1.3$.

San Diego, 2003), 540 pp.

3. W. Krolikowski and Yu.S. Kivshar, J. Opt. Soc. Am. B 13, 876 (1996).

4. A.B. Aceves, C. De Angelis, T. Peschel, R. Muschall, F. Lederer, S. Trillo, and S. Wabnitz, Phys. Rev. E 53, 1172 (1996).

5. R. Morandotti, H.S. Eisenberg, D. Mandelik, Y. Silberberg, D. Modotto, M. Sorel, C.R. Stanley, and J.S. Aitchison, Opt. Lett. 28, 834 (2003).

6. Yu.S. Kivshar, Phys. Rev. B 47, 11167 (1993).

7. A.A. Sukhorukov and Yu.S. Kivshar, Phys. Rev. Lett. 87, 083901 (2001).

8. F. Fedele, J. Yang, and Z. Chen, Opt. Lett. 30, 1506 (2005).

9. X. Wang, J. Young, Z. Chen, D. Weinstein and J. Yang. Opt. Express 14, 7362 (2006).

10. V.M. Apalkov and M.E. Raikh, Phys. Rev. Lett. 90, 253901 (2003).

11. E. Miyai, K. Sakai, T. Okano, W. Kunishi, D. Ohnishi, and S. Noda, Nature 441, 946 (2006).

12. K.G. Markis, S. Suntsov, D.N. Christodoulides, G.I. Stegeman, and A. Haché, Opt. Lett. 30, 2466 (2005).

13. M. Molina, R. Vicencio, and Yu.S. Kivshar, Opt. Lett. 31, 1693 (2006).

14. S. Darmanyan, A. Kobyakov, E. Schmidt, and F. Lederer, Phys. Rev. E 57, 3520 (1998). 